\title{
Existence of solutions for a coupled system of nonlinear fractional differential equations with fractional boundary conditions on the half-line
}

\author{
Yuji Liu', Bashir Ahmad²* and Ravi P Agarwal ${ }^{3,2}$
}

${ }^{*}$ Correspondence:

bashir_qau@yahoo.com

${ }^{2}$ Department of Mathematics,

Faculty of Science, King Abdulaziz

University, P.O. Box 80203, Jeddah,

21589, Saudi Arabia

Full list of author information is

available at the end of the article

\begin{abstract}
In this article, we study a boundary value problem of a coupled system of nonlinear Riemann-Liouville type fractional differential equations with fractional boundary conditions on the half-line. An appropriate compactness criterion is established to prove the existence of solutions of the problem by means of the Schauder fixed point theorem. An illustrative example is also given.

MSC: $34 \mathrm{~A} 08 ; 34 \mathrm{~A} 12 ; 34 \mathrm{~B} 40$
\end{abstract}

Keywords: boundary value problem; fractional differential system;

Riemann-Liouville fractional derivative; fixed-point theorem

\section{Introduction}

In recent years, the subject of fractional differential equations has gained a considerable attention and it has emerged as an interesting and popular field of research. It is mainly due to the fact that the tools of fractional calculus are found to be more practical and effective than the corresponding ones of classical calculus in the mathematical modeling of several phenomena involving fractals and chaos. In fact, fractional calculus has numerous applications in various disciplines of science and engineering such as mechanics, electricity, chemistry, biology, economics, control theory, signal and image processing, polymer rheology, regular variation in thermodynamics, biophysics, blood flow phenomena, aerodynamics, electro-dynamics of complex medium, viscoelasticity and damping, control theory, wave propagation, percolation, identification, fitting of experimental data, etc. For theoretical development and methods of solution for fractional differential equations, see the books [1-6] and references therein. For details on the geometric and physical interpretation of the derivatives of non-integer order, see [7-9]. Some recent results on fractional boundary value problems on a finite interval can be found in [10-21] and references therein.

In [10], using the monotone iterative method, Zhang investigated the existence and uniqueness of solutions for the following initial value problem of the fractional differential 
equations:

$$
\left\{\begin{array}{l}
D_{0^{+}}^{\alpha} u(t)=f(t, u(t)), \quad t \in(0, T], \\
\left.t^{1-\alpha} u(t)\right|_{t=0}=u_{0},
\end{array}\right.
$$

where $0<T<\infty$ and $D^{\alpha}$ is the Riemann-Liouville fractional derivative of order $\alpha \in(0,1)$. Arara et al. [22] studied the existence of bounded solutions for differential equations involving the Caputo fractional derivative on the unbounded domain given by

$$
\left\{\begin{array}{l}
{ }^{c} D_{0^{+}}^{\alpha} u(t)=f(t, u(t)), \quad t \in[0, \infty) \\
u(0)=u_{0} \\
u \text { is bounded on }[0, \infty)
\end{array}\right.
$$

where $\alpha \in(1,2),{ }^{c} D_{0^{+}}^{\alpha}$ is the Caputo fractional derivative of order $\alpha, u_{0} \in R$, and $f$ : $[0, \infty) \times R \rightarrow R$ is continuous. Using the Schauder fixed point theorem combined with the diagonalization method, it is proved that BVP (2) has at least one solution on $[0, \infty)$.

Zhao and Ge [23] considered the following boundary value problem for fractional differential equations:

$$
\left\{\begin{array}{l}
D_{0^{+}}^{\alpha} u(t)+f(t, u(t))=0, \quad 0<t<\infty, 1<\alpha<2 \\
u(0)=0 \\
\lim _{t \rightarrow \infty} D_{0^{+}}^{\alpha-1} u(t)=0
\end{array}\right.
$$

where $0<\xi<\infty, \beta \geq 0$ and $f$ is a given function, $D_{0^{+}}^{\alpha}$ is the Riemann-Liouville fractional derivative. By using the properties of the Green's function together with the Schauder fixed point theorem, it has been proved that BVP (3) has at least one positive solution subject to the assumptions: $f:[0, \infty) \times R \rightarrow[0, \infty)$ is continuous; and there exist a nondecreasing function $\omega \in C([0, \infty),[0, \infty))$ and a function $\phi \in L^{1}[0, \infty)$ such that $\left|f\left(t,\left(1+t^{\alpha-1}\right) u\right)\right| \leq$ $\phi(t) \omega(u)$ on $[0, \infty) \times[0, \infty)$.

In [24], Liu and Jia investigated the boundary value problem for a fractional differential equation of the form

$$
\left\{\begin{array}{l}
{ }^{c} D_{0^{+}}^{\alpha}\left[p(t) u^{\prime}(t)\right]+q(t) f(t, u(t))=0, \quad t>0, \\
p(0) u^{\prime}(0)=0, \\
\lim _{t \rightarrow \infty} u(t)=\int_{0}^{\infty} g(s) u(s) d s,
\end{array}\right.
$$

where ${ }^{c} D_{0^{+}}^{\alpha}$ is the Caputo fractional derivative of order $\alpha \in(0,1), f, g, p, q$ are given functions, $p(t)>0$ for all $t \geq 0$ with $\int_{0}^{\infty} \frac{1}{p(s)} d s<\infty$ and $k(s)=\int_{s}^{\infty} \frac{(r-s)^{\alpha-1}}{p(r)} d r$ being continuous on $[0, \infty), g \in L^{1}[0, \infty)$ with $\int_{0}^{\infty} g(s) d s<1$. The existence of at least three nonnegative solutions of the problem (4) was established by using fixed point theory and the method of upper and lower solutions.

For some more work on boundary value problems of fractional differential equations on a half-line/semi-infinite interval, we refer the reader to the papers [25-29].

On the other hand, the study for coupled systems of fractional differential equations is also important as such systems occur in various problems of applied nature; for instance, 
see [30-33]. Some recent results on coupled systems of fractional differential equations on a finite interval can be found in [34-37].

In this paper, we discuss the existence of solutions to a boundary value problem of a coupled system of nonlinear fractional differential equations on the half-line given by

$$
\left\{\begin{array}{l}
D_{0^{+}}^{\alpha} x(t)=f\left(t, y(t), D_{0^{+}}^{p} y(t)\right), \quad t \in(0, \infty), \\
D_{0^{+}}^{\beta} y(t)=g\left(t, x(t), D_{0^{+}}^{q} x(t)\right), \quad t \in(0, \infty), \\
a \lim _{t \rightarrow 0} t^{2-\alpha} x(t)-b \lim _{t \rightarrow 0} D_{0^{+}}^{\alpha-1} x(t)=x_{0}, \\
c \lim _{t \rightarrow 0} t^{2-\beta} y(t)-d \lim _{t \rightarrow 0} D_{0^{+}}^{\beta-1} x(t)=y_{0}, \\
\lim _{t \rightarrow \infty} D_{0^{+}}^{\alpha-1} x(t)=x_{1}, \\
\lim _{t \rightarrow \infty} D_{0^{+}}^{\beta-1} x(t)=y_{1},
\end{array}\right.
$$

where $a, b, c, d>0, \alpha, \beta \in(1,2), p \in(\beta-1, \beta), q \in(\alpha-1, \alpha), x_{0}, y_{0}, x_{1}, y_{1} \in R, D_{0^{+}}$is the standard Riemann-Liouville fractional derivative and $f, g:(0, \infty) \times R^{2} \rightarrow R$ are continuous functions and $f, g$ may be singular at $t=0$.

We establish sufficient conditions for the existence of solutions of (5) by applying the Schauder fixed point theorem. Our results are new in the sense that we consider BVP (5) on a half-line with the assumptions on $p, q$ of the form $p \in(\beta-1, \beta), q \in(\alpha-1, \alpha)$. Moreover, both the nonlinear functions $f$ and $g$ are allowed to be linear as well as super linear. The paper is organized as follows: the preliminary results are given in Section 2, the main results are presented in Section 3, while an example is discussed in Section 4 to illustrate the main theorems.

\section{Preliminary results}

Let us begin this section with some basic concepts of fractional calculus [1-3]. For $a>0$ and $b, c>0$, denote the gamma function and beta function respectively as

$$
\Gamma(a)=\int_{0}^{+\infty} s^{a-1} e^{-s} d s, \quad \mathbf{B}(b, c)=\int_{0}^{1}(1-x)^{b-1} x^{c-1} d x .
$$

Definition 2.1 The Riemann-Liouville fractional integral of order $\alpha>0$ of a continuous function $f:(0, \infty) \rightarrow R$ is given by

$$
I_{0^{+}}^{\alpha} f(t)=\frac{1}{\Gamma(\alpha)} \int_{0}^{t}(t-s)^{\alpha-1} f(s) d s
$$

provided that the right-hand side exists.

Definition 2.2 The Riemann-Liouville fractional derivative of order $\alpha>0$ of a continuous function $f:(0, \infty) \rightarrow R$ is given by

$$
D_{0^{+}}^{\alpha} f(t)=\frac{1}{\Gamma(n-\alpha)} \frac{d^{n+1}}{d t^{n+1}} \int_{0}^{t} \frac{f(s)}{(t-s)^{\alpha-n+1}} d s
$$

where $n-1<\alpha \leq n$, provided that the right-hand side is point-wise defined on $(0, \infty)$. 
It is easy to show that for $\varrho \geq 0$ and $\mu>-1$, we have

$$
I_{0^{+}}^{\varrho} t^{\mu}=\frac{\Gamma(\mu+1)}{\Gamma(\mu+\varrho+1)} t^{\mu+\varrho}, \quad D_{0^{0}}^{\varrho} t^{\mu}=\frac{\Gamma(\mu+1)}{\Gamma(\mu-\varrho+1)} t^{\mu-\varrho} .
$$

Let $C(0, \infty)$ be the set of all continuous functions on $(0, \infty)$. For $\sigma>\max \{q-\alpha, p-\beta\}$, ones sees from $p \in(\beta-1, \beta), q \in(\alpha-1, \alpha)$ that $\sigma>-1$. We choose

$$
X=\left\{\begin{array}{ll} 
& D_{0^{+}}^{q} \in C(0, \infty) \\
x \in C(0, \infty): & \frac{t^{2-\alpha}}{1+t^{\sigma+2}} x(t) \text { is bounded on }(0, \infty) \\
& \frac{t^{2+q-\alpha}}{1+t^{\sigma+2}} D_{0^{+}}^{q} x(t) \text { is bounded on }(0, \infty)
\end{array}\right\}
$$

and

$$
Y=\left\{\begin{array}{cl} 
& D_{0^{+}}^{p} y \in C(0, \infty) \\
y \in C(0, \infty): & \frac{t^{2-\beta}}{1+t^{\sigma+2}} x(t) \text { is bounded on }(0, \infty) \\
& \frac{t^{++p-\beta}}{1+t^{\sigma+2}} D_{0^{+}}^{p} x(t) \text { is bounded on }(0, \infty)
\end{array}\right\} .
$$

For $x \in X$, define the norm by

$$
\|x\|_{X}=\max \left\{\sup _{t \in(0, \infty)} \frac{t^{2-\alpha}}{1+t^{\sigma+2}}|x(t)|, \sup _{t \in(0, \infty)} \frac{t^{2+q-\alpha}}{1+t^{\sigma+2}}\left|D_{0^{+}}^{q} x(t)\right|\right\} .
$$

It is easy to show that $X$ is a real Banach space. For $y \in Y$, define the norm by

$$
\|y\|_{Y}=\max \left\{\sup _{t \in(0, \infty)} \frac{t^{2-\beta}}{1+t^{\sigma+2}}|y(t)|, \sup _{t \in(0, \infty)} \frac{t^{2+p-\beta}}{1+t^{\sigma+2}}\left|D_{0^{+}}^{p} y(t)\right|\right\} .
$$

It is easy to show that $Y$ is a real Banach space. Thus, $(X \times Y,\|\cdot\|)$ is Banach space with the norm defined by

$$
\|(x, y)\|=\max \left\{\|x\|_{X},\|y\|_{Y}\right\} \quad \text { for }(x, y) \in X \times Y .
$$

Lemma 2.1 Let $1<\alpha<2, x_{0}, x_{1} \in R$, and let $e:(0, \infty) \rightarrow R$ be a given function such that there exist numbers $M>0, \sigma>-1$ and $k>0$ with $|e(t)| \leq M t^{\sigma} e^{-k t}$. Then $x \in X$ is a solution of the problem

$$
\left\{\begin{array}{l}
D_{0^{+}}^{\alpha} x(t)=e(t), \quad t \in J=(0, \infty), \\
a \lim _{t \rightarrow 0} t^{2-\alpha} x(t)-b \lim _{t \rightarrow 0} D_{0^{+}}^{\alpha-1} x(t)=x_{0}, \\
\lim _{t \rightarrow \infty} D_{0^{+}}^{\alpha-1} x(t)=x_{1},
\end{array}\right.
$$

if and only if $x \in X$ and

$$
x(t)=\frac{1}{\Gamma(\alpha)} \int_{0}^{t}(t-s)^{\alpha-1} e(s) d s+\frac{x_{1}-\int_{0}^{\infty} e(s) d s}{\Gamma(\alpha)} t^{\alpha-1}+\frac{x_{0}+b x_{1}-b \int_{0}^{\infty} e(s) d s}{a} t^{\alpha-2} .
$$


Proof It is easy to see that $\int_{0}^{\infty} t^{\sigma} e^{-k t} d t<\infty$. For arbitrary constants $c_{1}, c_{2}$, the general solution of the equation $D_{0^{+}}^{\alpha} x(t)=e(t)$ can be written as

$$
x(t)=\frac{1}{\Gamma(\alpha)} \int_{0}^{t}(t-s)^{\alpha-1} e(s) d s+c_{1} t^{\alpha-1}+c_{2} t^{\alpha-2}
$$

with

$$
D_{0^{+}}^{\alpha-1} x(t)=\int_{0}^{t} e(s) d s+\Gamma(\alpha) c_{1}
$$

Using the boundary conditions of (6), we find that

$$
c_{1}=\frac{x_{1}-\int_{0}^{\infty} e(s) d s}{\Gamma(\alpha)}, \quad c_{2}=\frac{x_{0}+b x_{1}-b \int_{0}^{\infty} e(s) d s}{a} .
$$

Substituting the values of $c_{1}$ and $c_{2}$ in (8), we obtain (7).

Now, we prove $x \in X$. Clearly,

$$
\begin{aligned}
D_{0^{+}}^{q} x(t)= & \frac{\int_{0}^{t}(t-s)^{\alpha-q-1} e(s) d s}{\Gamma(\alpha-q)}+\frac{x_{1}-\int_{0}^{\infty} e(s) d s}{\Gamma(\alpha-q)} t^{\alpha-q-1} \\
& +\frac{x_{0}+b x_{1}-b \int_{0}^{\infty} e(s) d s}{a} \frac{\Gamma(\alpha-1) t^{\alpha-q-2}}{\Gamma(\alpha-q-1)} .
\end{aligned}
$$

It follows from (7) and (9) together with $|e(t)| \leq M t^{\sigma} e^{-k t}, \sigma>-1$ that $x, D_{0^{+}}^{q} x \in C(0, \infty)$.

Observe that

$$
\frac{t^{2-\alpha}}{1+t^{\sigma+2}}|x(t)| \leq \frac{\left|x_{0}+b x_{1}\right|}{a}+\frac{\left|x_{1}\right|}{\Gamma(\alpha)}+\frac{M}{\Gamma(\alpha)} \mathbf{B}(\alpha, \sigma+1)+\left(\frac{1}{\Gamma(\alpha)}+\frac{1}{a}\right) \frac{\Gamma(\sigma+1)}{k^{\sigma+1}}<+\infty
$$

and

$$
\begin{aligned}
\frac{t^{2+q-\alpha}}{1+t^{\sigma+2}}\left|D_{0^{+}}^{q} x(t)\right| \leq & \frac{M}{\Gamma(\alpha-q)} \frac{t^{\sigma+2}}{1+t^{\sigma+2}} \int_{0}^{1}(1-w)^{\alpha-q-1} w^{\sigma} d w \\
& +\frac{\left|x_{1}\right|}{\Gamma(\alpha-q)}+\frac{\left|x_{0}+b x_{1}\right|}{a} \frac{\Gamma(\alpha-1)}{|\Gamma(\alpha-q-1)|} \\
& +\left(\frac{1}{\Gamma(\alpha-q)}+\frac{\Gamma(\alpha-1)}{a|\Gamma(\alpha-q-1)|}\right) \frac{\Gamma(\sigma+1)}{k^{\sigma+1}}<+\infty .
\end{aligned}
$$

Hence $x \in X$.

Conversely, if $x \in X$ satisfies (7), then it can easily be shown that $x \in X$ and satisfies (6). This completes the proof.

Consider the coupled system of integral equations

$$
\left\{\begin{aligned}
x(t)= & \frac{1}{\Gamma(\alpha)} \int_{0}^{t}(t-s)^{\alpha-1} f\left(s, y(s), D_{0^{+}}^{p} y(s)\right) d s+\frac{x_{1}-\int_{0}^{\infty} f\left(s, y(s), D_{0^{+}}^{p} y(s)\right) d s}{\Gamma(\alpha)} t^{\alpha-1} \\
& +\frac{x_{0}+b x_{1}-\int_{0}^{\infty} f\left(s, y(s), D_{0^{+}}^{p} y(s)\right) d s}{a} t^{\alpha-2}, \\
y(t)= & \frac{1}{\Gamma(\beta)} \int_{0}^{t}(t-s)^{\alpha-1} g\left(s, x(s), D_{0^{+}}^{q} x(s)\right) d s+\frac{y_{1}-\int_{0}^{\infty} g\left(s, x(s), D_{0^{+}}^{q} x(s)\right) d s}{\Gamma(\alpha)} t^{\beta-1} \\
& +\frac{y_{0}+d y_{1}-\int_{0}^{\infty} g\left(s, x(s), D_{0^{+}}^{q} x(s)\right) d s}{c} t^{\beta-2} .
\end{aligned}\right.
$$


For the sequel, we need the following assumptions:

(H) There exist numbers $\sigma_{i}, \mu_{i} \in(-1, \sigma), k_{i}>0, l_{i}>0(i=0,1,2)$ and positive numbers $A, B, C, A_{1}, B_{1}, C_{1}$ such that for $t \in(0, \infty), u_{1}, u_{2}, v_{1}, v_{2} \in R, f$ and $g$ satisfy the conditions

$$
\left|f\left(t, \frac{1+t^{\sigma+2}}{t^{2-\beta}} u_{1}, \frac{1+t^{\sigma+2}}{t^{2+p-\beta}} u_{2}\right)-C t^{\sigma_{0}} e^{-k_{0} t}\right| \leq A t^{\sigma_{1}} e^{-k_{1} t}\left|u_{1}\right|+B t^{\sigma_{2}} e^{-k_{2} t}\left|u_{2}\right|
$$

and

$$
\left|g\left(t, \frac{1+t^{\sigma+2}}{t^{2-\alpha}} v_{1}, \frac{1+t^{\sigma+2}}{t^{2+q-\alpha}} v_{2}\right)-C_{1} t^{\mu_{0}} e^{-l_{0} t}\right| \leq A_{1} t^{\mu_{1}} e^{-l_{1} t}\left|v_{1}\right|+B_{1} t^{\mu_{2}} e^{-l_{2} t}\left|v_{2}\right| ;
$$

(G) There exist numbers $\sigma_{i}, \mu_{i} \in(-1, \sigma), k_{i}>0, l_{i}>0(i=0,1,2), \delta>1$ and positive numbers $A, B, C, A_{1}, B_{1}, C_{1}$ such that for $t \in(0, \infty), u_{1}, u_{2}, v_{1}, v_{2} \in R, f$ and $g$ satisfy the conditions

$$
\left|f\left(t, \frac{1+t^{\sigma+2}}{t^{2-\beta}} u_{1}, \frac{1+t^{\sigma+2}}{t^{2+p-\beta}} u_{2}\right)-C t^{\sigma_{0}} e^{-k_{0} t}\right| \leq A t^{\sigma_{1}} e^{-k_{1} t}\left|u_{1}\right|^{\delta}+B t^{\sigma_{2}} e^{-k_{2} t}\left|u_{2}\right|^{\delta}
$$

and

$$
\left|g\left(t, \frac{1+t^{\sigma+2}}{t^{2-\alpha}} v_{1}, \frac{1+t^{\sigma+2}}{t^{2+q-\alpha}} v_{2}\right)-C_{1} t^{\mu_{0}} e^{-l_{0} t}\right| \leq A_{1} t^{\mu_{1}} e^{-l_{1} t}\left|v_{1}\right|^{\delta}+B_{1} t^{\mu_{2}} e^{-l_{2} t}\left|v_{2}\right|^{\delta} .
$$

Lemma 2.2 Suppose that (H) or (G) holds. Then $(x, y) \in X \times Y$ is a solution of (5) if and only if $(x, y) \in X \times Y$ is a solution of $(10)$.

Proof Let $(x, y) \in X \times Y$. In view of the assumption $(\mathrm{H})$, it follows that

$$
\begin{aligned}
\left|f\left(t, y(t), D_{0^{+}}^{p} y(t)\right)\right| & =\left|f\left(t, \frac{1+t^{\sigma+2}}{t^{2-\beta}} \frac{t^{2-\beta}}{1+t^{\sigma+2}} y(t), \frac{1+t^{\sigma+2}}{t^{2+p-\beta}} \frac{t^{2+p-\beta}}{1+t^{\sigma+2}} D_{0^{+}}^{p} y(t)\right)\right| \\
& \leq C t^{\sigma_{0}} e^{-k_{0} t}+A t^{\sigma_{1}} e^{-k_{1} t}\|y\|_{Y}+B t^{\sigma_{2}} e^{-k_{2} t}\|y\|_{Y}
\end{aligned}
$$

and

$$
\left|g\left(t, x(t), D_{0^{+}}^{q} p x(t)\right)\right| \leq C_{1} t^{\mu_{0}} e^{-l_{0} t}+A_{1} t^{\mu_{1}} e^{-l_{1} t}\|x\|_{X}+B_{1} t^{\mu_{2}} e^{-l_{2} t}\|x\|_{X} .
$$

The rest of the proof follows from Lemma 2.1. Similarly, we can show that the result holds if $(\mathrm{G})$ holds. This completes the proof.

Let us define an operator $F: X \times Y \rightarrow X \times Y$ as

$$
F(x, y)(t)=\left(\left(F_{1} y\right)(t),\left(F_{2} x\right)(t)\right)
$$

where

$$
\begin{aligned}
\left(F_{1} y\right)(t)= & \frac{1}{\Gamma(\alpha)} \int_{0}^{t}(t-s)^{\alpha-1} f\left(s, y(s), D_{0^{+}}^{p} y(s)\right) d s+\frac{x_{1}-\int_{0}^{\infty} f\left(s, y(s), D_{0^{+}}^{p} y(s)\right) d s}{\Gamma(\alpha)} t^{\alpha-1} \\
& +\frac{x_{0}+b x_{1}-\int_{0}^{\infty} f\left(s, y(s), D_{0^{+}}^{p} y(s)\right) d s}{a} t^{\alpha-2}
\end{aligned}
$$


and

$$
\begin{aligned}
\left(F_{2} x\right)(t)= & \frac{1}{\Gamma(\beta)} \int_{0}^{t}(t-s)^{\alpha-1} g\left(s, x(s), D_{0^{+}}^{q} x(s)\right) d s+\frac{y_{1}-\int_{0}^{\infty} g\left(s, x(s), D_{0^{+}}^{q} x(s)\right) d s}{\Gamma(\alpha)} t^{\beta-1} \\
& +\frac{y_{0}+d y_{1}-\int_{0}^{\infty} g\left(s, x(s), D_{0^{+}}^{q} x(s)\right) d s}{c} t^{\beta-2} .
\end{aligned}
$$

Lemma 2.3 Suppose that $(\mathrm{H})$ or $(\mathrm{G})$ holds. Then the fixed point of the operator $F$ coincides with the solution of (5) and $F: X \times Y \rightarrow X \times Y$ is completely continuous.

Proof It follows from Lemma 2.2 that the fixed point of the operator $F$ coincides with the solution of (5). Suppose that $(\mathrm{H})$ holds. The remaining proof consists of the following five steps.

Step 1. We show that $F: X \times Y \rightarrow X \times Y$ is well defined and maps bounded sets into bounded sets.

$$
\begin{aligned}
& \text { For }(x, y) \in X \times Y \text {, we get } \\
& \qquad \begin{aligned}
r= & \max \left\{\|x\|_{X},\|y\|_{Y}\right\} \\
& =\max \left\{\sup _{t \in(0, \infty)} \frac{t^{2-\alpha}}{1+t^{\sigma+2}}|x(t)|, \sup _{t \in(0, \infty)} \frac{t^{2-\beta}}{1+t^{\sigma+2}}|y(t)|,\right. \\
& \left.\sup _{t \in(0, \infty)} \frac{t^{2+q-\alpha}}{1+t^{\sigma+2}}\left|D_{0^{+}}^{q} x(t)\right|, \sup _{t \in(0, \infty)} \frac{t^{2+p-\beta}}{1+t^{\sigma+2}}\left|D_{0^{+}}^{p} y(t)\right|\right\}<\infty .
\end{aligned}
\end{aligned}
$$

By the definition of $F$, we have

$$
\left(F_{1} y\right), D_{0^{+}}^{q}\left(F_{1} y\right) \in C(0, \infty)
$$

By the method used in Lemma 2.2, we get $(\mathrm{H})$ implies that

$$
\begin{aligned}
& \left|f\left(t, y(t), D_{0^{+}}^{p} y(t)\right)\right| \\
& \quad=\left|f\left(t, \frac{1+t^{\sigma+2}}{t^{2-\beta}} \frac{t^{2-\beta}}{1+t^{\sigma+2}} y(t), \frac{1+t^{\sigma+2}}{t^{2+p-\beta}} \frac{t^{2+p-\beta}}{1+t^{\sigma+2}} D_{0^{+}}^{p} y(t)\right)\right| \\
& \quad \leq C t^{\sigma_{0}} e^{-k_{0} t}+A t^{\sigma_{1}} e^{-k_{1} t}\left|\frac{t^{2-\beta}}{1+t^{\sigma+2}} y(t)\right|+B t^{\sigma_{2}} e^{-k_{2} t}\left|\frac{t^{2+p-\beta}}{1+t^{\sigma+2}} D_{0^{+}}^{p} y(t)\right| \\
& \quad \leq C t^{\sigma_{0}} e^{-k_{0} t}+A r t^{\sigma_{1}} e^{-k_{1} t}+B r t^{\sigma_{2}} e^{-k_{2} t}
\end{aligned}
$$

and

$$
\left|g\left(t, x(t), D_{0^{+}}^{q} x(t)\right)\right| \leq C_{1} t^{\mu_{0}} e^{-l_{0} t}+A_{1} r t^{\mu_{1}} e^{-l_{1} t}+B_{1} r t^{\mu_{2}} e^{-l_{2} t}
$$

Hence

$$
\begin{aligned}
& \frac{t^{2-\alpha}}{1+t^{\sigma+2}}\left|\left(F_{1} y\right)(t)\right| \\
& \quad \leq \frac{1}{\Gamma(\alpha)} C \int_{0}^{1}(1-w)^{\alpha-1} w^{\sigma_{0}} d w+A r \int_{0}^{1}(1-w)^{\alpha-1} w^{\sigma_{1}} d w
\end{aligned}
$$


Lu et al. Advances in Difference Equations 2013, 2013:46

Page 8 of 19

http://www.advancesindifferenceequations.com/content/2013/1/46

$$
\begin{aligned}
& +B r \int_{0}^{1}(1-w)^{\alpha-1} w^{\sigma_{2}} d w+\frac{\left|x_{1}\right|}{\Gamma(\alpha)}+\frac{\left|x_{0}+b x_{1}\right|}{a} \\
& +\left(\frac{1}{\Gamma(\alpha)}+\frac{1}{a}\right)\left[\frac{C}{k_{0}^{\sigma_{0}+1}} \Gamma\left(\sigma_{0}+1\right)+\frac{A r}{k_{1}^{\sigma_{1}+1}} \Gamma\left(\sigma_{1}+1\right)+\frac{B r}{k_{2}^{\sigma_{2}+1}} \Gamma\left(\sigma_{2}+1\right)\right]<+\infty .
\end{aligned}
$$

Furthermore, we have

$$
\begin{aligned}
D_{0^{+}}^{q}\left(F_{1} y\right)(t)= & \frac{\int_{0}^{t}(t-s)^{\alpha-q-1} f\left(s, y(s), D_{0^{+}}^{p} y(s)\right) d s}{\Gamma(\alpha-q)} \\
& +\left(x_{1}-\int_{0}^{\infty} f\left(s, y(s), D_{0^{+}}^{p} y(s)\right) d s\right) \frac{t^{\alpha-q-1}}{\Gamma(\alpha-q)} \\
& +\left(x_{0}+b x_{1}-\int_{0}^{\infty} f\left(s, y(s), D_{0^{+}}^{p} y(s)\right) d s\right) \frac{\Gamma(\alpha-1) t^{\alpha-q-2}}{\Gamma(\alpha-q-1)} .
\end{aligned}
$$

Similarly, we obtain

$$
\begin{aligned}
& \frac{t^{2+q-\alpha}}{1+t^{\sigma+2}}\left|D_{0^{+}}^{q}\left(F_{1} y\right)(t)\right| \\
& \leq \frac{1}{\Gamma(\alpha-q)} C \int_{0}^{1}(1-w)^{\alpha-p-1} w^{\sigma_{0}} d w+A r \int_{0}^{1}(1-w)^{\alpha-q-1} w^{\sigma_{1}} d w \\
& +B r \int_{0}^{1}(1-w)^{\alpha-q-1} w^{\sigma_{2}} d w+\frac{\left|x_{1}\right|}{\Gamma(\alpha-q)}+\frac{\left|x_{0}+b x_{1}\right| \Gamma(\alpha-1)}{|\Gamma(\alpha-q-1)|} \\
& +\left(\frac{1}{\Gamma(\alpha-q)} \frac{t}{1+t^{\sigma+2}}+\frac{\Gamma(\alpha-q)}{|\Gamma(\alpha-q-1)|} \frac{1}{1+t^{\sigma+2}}\right) \\
& \quad \times\left[\frac{C}{k_{0}^{\sigma_{0}+1}} \Gamma\left(\sigma_{0}+1\right)+\frac{A r}{k_{1}^{\sigma_{1}+1}} \Gamma\left(\sigma_{1}+1\right)+\frac{B r}{k_{2}^{\sigma_{2}+1}} \Gamma\left(\sigma_{2}+1\right)\right]<+\infty .
\end{aligned}
$$

Then $F_{1} y \in X$. Similarly, we can prove that $F_{2} x \in Y$. Thus $F: X \times Y \rightarrow X \times Y$ is well defined. It is easy to show similarly that $F$ maps bounded sets into bounded sets.

Step 2. We show that $F$ is continuous.

Let $\left(u_{n}, v_{n}\right) \in X \times Y$ with $\left(u_{n}, v_{n}\right) \rightarrow\left(u_{0}, v_{0}\right)$ as $n \rightarrow \infty$. We will prove that $\left(F_{1} v_{n}, F_{2} u_{n}\right) \rightarrow$ $\left(F_{1} v_{0}, F_{2} u_{0}\right)$ as $n \rightarrow \infty$. It is easy to see that there exists $r>0$ such that

$$
\begin{aligned}
\left\|\left(u_{n}, v_{n}\right)\right\|= & \max \left\{\left\|u_{n}\right\|_{X},\left\|v_{n}\right\|_{Y}\right\} \\
= & \max \left\{\sup _{t \in(0, \infty} \frac{t^{2-\alpha}}{1+t^{\sigma+2}}\left|u_{n}(t)\right|, \sup _{t \in(0, \infty} \frac{t^{2-\beta}}{1+t^{\sigma+2}}\left|v_{n}(t)\right|,\right. \\
& \left.\sup _{t \in(0, \infty)} \frac{t^{2+q-\alpha}}{1+t^{\sigma+2}}\left|D_{0^{+}}^{q} u_{n}(t)\right|, \sup _{t \in(0, \infty)} \frac{t^{2+p-\beta}}{1+t^{\sigma+2}}\left|D_{0^{+}}^{p} v_{n}(t)\right|\right\} \leq r<\infty .
\end{aligned}
$$

Then $(\mathrm{H})$ implies that

$$
\begin{aligned}
\left|f\left(t, v_{n}(t), D_{0^{+}}^{p} v_{n}(t)\right)\right| & =\left|f\left(t, \frac{1+t^{\sigma+2}}{t^{2-\beta}} \frac{t^{2-\beta}}{1+t^{\sigma+2}} v_{n}(t), \frac{1+t^{\sigma+2}}{t^{2+p-\beta}} \frac{t^{2+p-\beta}}{1+t^{\sigma+2}} D_{0^{+}}^{p} v_{n}(t)\right)\right| \\
& \leq C t^{\sigma_{0}} e^{-k_{0} t}+A r t^{\sigma_{1}} e^{-k_{1} t}+B r t^{\sigma_{2}} e^{-k_{2} t}
\end{aligned}
$$


and

$$
\left|g\left(t, u_{n}(t), D_{0^{+}}^{q} u_{n}(t)\right)\right| \leq C_{1} t^{\mu_{0}} e^{-l_{0} t}+A_{1} r t^{\mu_{1}} e^{-l_{1} t}+B_{1} r t^{\mu_{2}} e^{-l_{2} t} .
$$

Observe that

$$
\begin{aligned}
\left(F_{1} v_{n}\right)(t)= & \frac{1}{\Gamma(\alpha)} \int_{0}^{t}(t-s)^{\alpha-1} f\left(s, v_{n}(s), D_{0^{+}}^{p} v_{n}(s)\right) d s \\
& +\frac{x_{1}-\int_{0}^{\infty} f\left(s, v_{n}(s), D_{0^{+}}^{p} v_{n}(s)\right) d s}{\Gamma(\alpha)} t^{\alpha-1} \\
& +\frac{x_{0}+b x_{1}-\int_{0}^{\infty} f\left(s, v_{n}(s), D_{0^{+}}^{p} v_{n}(s)\right) d s}{a} t^{\alpha-2}, \\
\left(F_{2} u_{n}\right)(t)= & \frac{1}{\Gamma(\beta)} \int_{0}^{t}(t-s)^{\alpha-1} g\left(s, u_{n}(s), D_{0^{+}}^{q} u_{n}(s)\right) d s \\
& +\frac{y_{1}-\int_{0}^{\infty} g\left(s, u_{n}(s), D_{0^{+}}^{p} u_{n}(s)\right) d s}{\Gamma(\alpha)} t^{\beta-1} \\
& +\frac{y_{0}+d y_{1}-\int_{0}^{\infty} g\left(s, u_{n}(s), D_{0^{+}}^{p} u_{n}(s)\right) d s}{c} t^{\beta-2} .
\end{aligned}
$$

Hence

$$
\begin{aligned}
& \frac{t^{2-\alpha}}{1+t^{\sigma+2}}\left|\left(F_{1} v_{n}\right)(t)-\left(F_{1} v_{0}\right)(t)\right| \\
& \leq \frac{2}{\Gamma(\alpha)} C \int_{0}^{1}(1-w)^{\alpha-1} w^{\sigma_{0}} d w+2 A r \int_{0}^{1}(1-w)^{\alpha-1} w^{\sigma_{1}} d w+2 B r \int_{0}^{1}(1-w)^{\alpha-1} w^{\sigma_{2}} d w \\
& \quad+\left(\frac{1}{\Gamma(\alpha)}+\frac{1}{a}\right)\left[\frac{C}{k_{0}^{\sigma_{0}+1}} \Gamma\left(\sigma_{0}+1\right)+\frac{A r}{k_{1}^{\sigma_{1}+1}} \Gamma\left(\sigma_{1}+1\right)+\frac{B r}{k_{2}^{\sigma_{2}+1}} \Gamma\left(\sigma_{2}+1\right)\right]<+\infty .
\end{aligned}
$$

It follows from the Lebesgue dominated convergence theorem that

$$
\lim _{n \rightarrow \infty} \frac{t^{2-\alpha}}{1+t^{\sigma+2}}\left|\left(F_{1} v_{n}\right)(t)-\left(F_{1} v_{0}\right)(t)\right|=0
$$

Furthermore, we have

$$
\begin{aligned}
D_{0^{+}}^{q}\left(F_{1} v_{n}\right)(t)= & \frac{\int_{0}^{t}(t-s)^{\alpha-q-1} f\left(s, v_{n}(s), D_{0^{+}}^{p} v_{n}(s)\right) d s}{\Gamma(\alpha-q)} \\
& +\left(x_{1}-\int_{0}^{\infty} f\left(s, v_{n}(s), D_{0^{+}}^{p} v_{n}(s)\right) d s\right) \frac{t^{\alpha-q-1}}{\Gamma(\alpha-q)} \\
& +\left(x_{0}+b x_{1}-\int_{0}^{\infty} f\left(s, v_{n}(s), D_{0^{+}}^{p} v_{n}(s)\right) d s\right) \frac{\Gamma(\alpha-1) t^{\alpha-q-2}}{\Gamma(\alpha-q-1)} .
\end{aligned}
$$

In a similar manner, we find that

$$
\lim _{n \rightarrow \infty} \frac{t^{2+q-\alpha}}{1+t^{\sigma+2}}\left|D_{0^{+}}^{q}\left(F_{1} v_{n}\right)(t)-D_{0^{+}}^{q}\left(F_{1} v_{0}\right)(t)\right|=0
$$


Thus,

$$
\begin{aligned}
& \lim _{n \rightarrow \infty} \frac{t^{2-\beta}}{1+t^{\sigma+2}}\left|\left(F_{2} u_{n}\right)(t)-\left(F_{2} u_{0}\right)(t)\right|=0, \\
& \lim _{n \rightarrow \infty} \frac{t^{2+p-\eta}}{1+t^{\sigma+2}}\left|D_{0^{+}}^{p}\left(F_{2} u_{n}\right)(t)-D_{0^{+}}^{p}\left(F_{2} u_{0}\right)(t)\right|=0 .
\end{aligned}
$$

Hence we get

$$
\lim _{n \rightarrow \infty}\left(F_{1} v_{n}, F_{2} u_{n}\right)=\left(F_{1} v_{0}, F_{2} u_{0}\right)
$$

which shows that $F$ is continuous.

In order to show that $F$ maps bounded sets of $X \times Y$ to relatively compact sets of $X \times Y$, it suffices to prove that both $F_{1}$ and $F_{2}$ map bounded sets to relatively compact sets.

Recall $W \subset X$ is relatively compact if

(i) it is bounded,

(ii) both $\frac{t^{2-\alpha}}{1+t^{\sigma+2}} W$ and $\frac{t^{2+q-\alpha}}{1+t^{\sigma+2}} W$ are equicontinuous on any closed subinterval $[a, b]$ of $(0, \infty)$

(iii) both $\frac{t^{2-\alpha}}{1+t^{\sigma+2}} W$ and $\frac{t^{2+q-\alpha}}{1+t^{\sigma+2}} W$ are equiconvergent at $t=0$,

(iv) both $\frac{t^{2-\alpha}}{1+t^{\sigma+2}} W$ and $\frac{t^{2+q-\alpha}}{1+t^{\sigma+2}} W$ are equiconvergent at $t=\infty$.

$W \subset Y$ is relatively compact if

(i) it is bounded,

(ii) both $\frac{t^{2-\beta}}{1+t^{\sigma+2}} W$ and $\frac{t^{2+p-\beta}}{1+t^{\sigma+2}} W$ are equicontinuous on any closed subinterval $[a, b]$ of $(0, \infty)$

(iii) both $\frac{t^{2-\beta}}{1+t^{\sigma+2}} W$ and $\frac{t^{2+p-\beta}}{1+t^{\sigma+2}} W$ are equiconvergent at $t=0$,

(iv) both $\frac{t^{2-\beta}}{1+t^{\sigma+2}} W$ and $\frac{t^{2+p-\beta}}{1+t^{\sigma+2}} W$ are equiconvergent at $t=\infty$.

Step 3. We prove that both $F_{1}: \Omega_{1} \rightarrow Y$ and $F_{2}: \Omega_{2} \rightarrow X$ are equicontinuous on a finite closed interval of $(0, \infty)$.

Let $\Omega_{1} \subset Y$ and $\Omega_{2} \subset X$ be bounded sets. Then there exists $r>0$ such that

$$
\begin{aligned}
\|(u, v)\|= & \max \left\{\|u\|_{X},\|v\|_{Y}\right\} \\
= & \max \left\{\sup _{t \in(0, \infty} \frac{t^{2-\alpha}}{1+t^{\sigma+2}}|u(t)|, \sup _{t \in(0, \infty} \frac{t^{2-\beta}}{1+t^{\sigma+2}}|v(t)|,\right. \\
& \left.\sup _{t \in(0, \infty)} \frac{t^{2+q-\alpha}}{1+t^{\sigma+2}}\left|D_{0^{+}}^{q} u(t)\right|, \sup _{t \in(0, \infty)} \frac{t^{2+p-\beta}}{1+t^{\sigma+2}}\left|D_{0^{+}}^{p} v(t)\right|\right\} \\
\leq & r<\infty, \quad u \in \Omega_{2}, v \in \Omega_{1} .
\end{aligned}
$$

Then $(\mathrm{H})$ implies that

$$
\left|f\left(t, v(t), D_{0^{+}}^{p} \nu(t)\right)\right| \leq C t^{\sigma_{0}} e^{-k_{0} t}+A r t^{\sigma_{1}} e^{-k_{1} t}+B r t^{\sigma_{2}} e^{-k_{2} t}
$$

and

$$
\left|g\left(t, u(t), D_{0^{+}}^{q} u(t)\right)\right| \leq C_{1} t^{\mu_{0}} e^{-l_{0} t}+A_{1} r t^{\mu_{1}} e^{-l_{1} t}+B_{1} r t^{\mu_{2}} e^{-l_{2} t}
$$


Lu et al. Advances in Difference Equations 2013, 2013:46

Page 11 of 19

http://www.advancesindifferenceequations.com/content/2013/1/46

For $[a, b] \subset(0, \infty)$ with $t_{1}, t_{2} \in[a, b]$ with $t_{1}<t_{2}$ and $v \in \Omega_{1}$, we have

$$
\begin{aligned}
& \left|\frac{t_{1}^{2-\alpha}}{1+t_{1}^{\sigma+2}}\left(F_{1} v\right)\left(t_{1}\right)-\frac{t_{2}^{2-\alpha}}{1+t_{2}^{\sigma+2}}\left(F_{1} v\right)\left(t_{2}\right)\right| \\
& \leq \frac{1}{\Gamma(\alpha)} \mid \frac{t_{1}^{2-\alpha}}{1+t_{1}^{\sigma+2}} \int_{0}^{t_{1}}\left(t_{1}-s\right)^{\alpha-1} f\left(s, v(s), D_{0^{+}}^{p} v(s)\right) d s \\
& \quad-\frac{t_{2}^{2-\alpha}}{1+t_{2}^{\sigma+2}} \int_{0}^{t_{2}}\left(t_{2}-s\right)^{\alpha-1} f\left(s, v(s), D_{0^{+}}^{p} v(s)\right) d s \mid \\
& \quad+\frac{\left|x_{1}-\int_{0}^{\infty} f\left(s, v(s), D_{0^{+}}^{p} v(s)\right) d s\right|}{\Gamma(\alpha)}\left|\frac{t_{1}}{1+t_{1}^{\sigma+2}}-\frac{t_{2}}{1+t_{2}^{\sigma+2}}\right| \\
& \quad+\left|x_{0}+b x_{1}-\int_{0}^{\infty} f\left(s, v(s), D_{0^{+}}^{p} v(s)\right) d s\right|\left|\frac{1}{1+t_{1}^{\sigma+2}}-\frac{1}{1+t_{2}^{\sigma+2}}\right| .
\end{aligned}
$$

Since $\left|a^{v}-b^{v}\right| \leq|a-b|^{v}$ for all $a, b \geq 0$ and $v \in(0,1)$, therefore, we get

$$
\begin{aligned}
& \mid \frac{t_{1}^{2-\alpha}}{1+t_{1}^{\sigma+2}} \int_{0}^{t_{1}}\left(t_{1}-s\right)^{\alpha-1} f\left(s, v(s), D_{0^{+}}^{p} v(s)\right) d s \\
& \quad-\frac{t_{2}^{2-\alpha}}{1+t_{2}^{\sigma+2}} \int_{0}^{t_{2}}\left(t_{2}-s\right)^{\alpha-1} f\left(s, v(s), D_{0^{+}}^{p} v(s)\right) d s \mid \\
& \leq\left|\frac{t_{1}^{2-\alpha}}{1+t_{1}^{\sigma+2}}-\frac{t_{2}^{2-\alpha}}{1+t_{2}^{\sigma+2}}\right| \\
& \quad \times\left[C b^{\alpha+\sigma_{0}} \int_{0}^{1}(1-w)^{\alpha-1} w^{\sigma_{0}} d w+A r b^{\alpha+\sigma_{1}} \int_{0}^{1}(1-w)^{\alpha-1} w^{\sigma_{1}} d w\right. \\
& \left.+B r b^{\alpha+\sigma_{2}} \int_{0}^{1}(1-w)^{\alpha-1} w^{\sigma_{2}} d w\right] \\
& \quad+C b^{\alpha+\sigma_{0}} \int_{t_{1} / t_{2}}^{1}(1-w)^{\alpha-1} w^{\sigma_{0}} d w+A r b^{\alpha+\sigma_{1}} \int_{t_{1} / t_{2}}^{1}(1-w)^{\alpha-1} w^{\sigma_{1}} d w \\
& +B r b^{\alpha+\sigma_{2}} \int_{t_{1} / t_{2}}^{1}(1-w)^{\alpha-1} w^{\sigma_{2}} d w+\left|t_{1}-t_{2}\right|^{\alpha-1}\left[\frac{C b^{\sigma_{0}+1}}{\sigma_{0}+1}+\frac{A r b^{\sigma_{1}+1}}{\sigma+1}+\frac{B r b^{\sigma_{2}+1}}{\sigma_{2}+1}\right]
\end{aligned}
$$

$\rightarrow 0 \quad$ uniformly in $\Omega_{1}$ as $t_{2} \rightarrow t_{1}$.

Moreover, we have

$$
\begin{aligned}
& \left|x_{1}-\int_{0}^{\infty} f\left(s, v(s), D_{0^{+}}^{p} v(s)\right) d s\right| \\
& \quad \leq\left|x_{1}\right|+\frac{C}{k_{0}^{\sigma_{0}+1}} \Gamma\left(\sigma_{0}+1\right)+\frac{A r}{k_{1}^{\sigma_{1}+1}} \Gamma\left(\sigma_{1}+1\right)+\frac{B r}{k_{2}^{\sigma_{2}+1}} \Gamma\left(\sigma_{2}+1\right) .
\end{aligned}
$$

Thus,

$$
\begin{aligned}
& \left|x_{0}+b x_{1}-\int_{0}^{\infty} f\left(s, v(s), D_{0^{+}}^{p} v(s)\right) d s\right| \\
& \quad \leq\left|x_{0}+b x_{1}\right|+\frac{C \Gamma\left(\sigma_{0}+1\right)}{k_{0}^{\sigma_{0}+1}}+\frac{\operatorname{Ar} \Gamma\left(\sigma_{1}+1\right)}{k_{1}^{\sigma_{1}+1}}+\frac{\operatorname{Br} \Gamma\left(\sigma_{2}+1\right)}{k_{2}^{\sigma_{2}+1}} .
\end{aligned}
$$


Hence

$$
\left|\frac{t_{1}^{2-\alpha}}{1+t_{1}^{\sigma+2}}\left(F_{1} v\right)\left(t_{1}\right)-\frac{t_{2}^{2-\alpha}}{1+t_{2}^{\sigma+2}}\left(F_{1} v\right)\left(t_{2}\right)\right| \rightarrow 0 \quad \text { uniformly in } \Omega_{1} \text { as } t_{2} \rightarrow t_{1} \text {. }
$$

On the other hand, we have

$$
\begin{aligned}
& \left|\frac{t_{1}^{2+q-\alpha}}{1+t_{1}^{\sigma+2}} D_{0^{+}}^{q}\left(F_{1} v\right)\left(t_{1}\right)-\frac{t_{2}^{2+q-\alpha}}{1+t_{2}^{\sigma+2}} D_{0^{+}}^{q}\left(F_{1} v\right)\left(t_{2}\right)\right| \\
& \leq \frac{1}{\Gamma(\alpha-q)} \mid \frac{t_{1}^{2+q-\alpha}}{1+t_{1}^{\sigma+2}} \int_{0}^{t_{1}}\left(t_{1}-s\right)^{\alpha-q-1} f\left(s, v(s), D_{0^{+}}^{p} v(s)\right) d s \\
& \quad-\frac{t_{2}^{2+q-\alpha}}{1+t_{2}^{\sigma+2}} \int_{0}^{t_{2}}\left(t_{2}-s\right)^{\alpha-q-1} f\left(s, v(s), D_{0^{+}}^{p} v(s)\right) d s \mid \\
& \quad+\frac{\left|x_{1}-\int_{0}^{\infty} f\left(s, v(s), D_{0^{+}}^{p} v(s)\right) d s\right|}{\Gamma(\alpha-q)}\left|\frac{t_{1}}{1+t_{1}^{\sigma+2}}-\frac{t_{2}}{1+t_{2}^{\sigma+2}}\right| \\
& \quad+\left|x_{0}+b x_{1}-\int_{0}^{\infty} f\left(s, v(s), D_{0^{+}}^{p} v(s)\right) d s\right| \frac{\Gamma(\alpha-1)}{|\Gamma(\alpha-q-1)|}\left|\frac{1}{1+t_{1}^{\sigma+2}}-\frac{1}{1+t_{2}^{\sigma+2}}\right| .
\end{aligned}
$$

Let

$$
M_{0}=\max \left\{a^{\alpha-q+\sigma_{0}}, b^{\alpha-q+\sigma_{0}}, a^{\alpha-q+\sigma_{1}}, b^{\alpha-q+\sigma_{1}}, a^{\alpha-q+\sigma_{2}}, b^{\alpha-q+\sigma_{2}}\right\} .
$$

Note that $q \in(\alpha-1, \alpha-1 / 2)$ and $\left|a^{\nu}-b^{v}\right| \leq|a-b|^{\nu}$ for all $a, b \geq 0$ and $v \in(0,1)$. Note $\sigma>\max \{q-\alpha, p-\beta\}$. Then

$$
\begin{aligned}
& \mid \frac{t_{1}^{2+q-\alpha}}{1+t_{1}^{\sigma+2}} \int_{0}^{t_{1}}\left(t_{1}-s\right)^{\alpha-q-1} f\left(s, v(s), D_{0^{+}}^{p} v(s)\right) d s \\
& \quad-\frac{t_{2}^{2+q-\alpha}}{1+t_{2}^{\sigma+2}} \int_{0}^{t_{2}}\left(t_{2}-s\right)^{\alpha-q-1} f\left(s, v(s), D_{0^{+}}^{p} v(s)\right) d s \mid \\
& \leq M_{0}\left|\frac{t_{1}^{2+q-\alpha}}{1+t_{1}^{\sigma+2}}-\frac{t_{2}^{2+q-\alpha}}{1+t_{2}^{\sigma+2}}\right| \\
& \quad \times\left[C \mathbf{B}\left(\alpha-q, \sigma_{0}+1\right)+A r \mathbf{B}\left(\alpha-q, \sigma_{1}+1\right)+B r \mathbf{B}\left(\alpha-q, \sigma_{2}+1\right)\right] \\
& \quad+M_{0}\left[C \int_{t_{1} / t_{2}}^{1}(1-w)^{\alpha-q-1} w^{\sigma_{0}} d w+A r \int_{t_{1} / t_{2}}^{1}(1-w)^{\alpha-q-1} w^{\sigma_{1}} d w\right. \\
& \left.\quad+B r \int_{t_{1} / t_{2}}^{1}(1-w)^{\alpha-q-1} w^{\sigma_{2}} d w\right] \\
& \quad+\left|t_{2}^{\alpha+\sigma_{0}-q}-t_{1}^{\alpha+\sigma_{0}-q}\right|\left[C \mathbf{B}\left(\alpha-q, \sigma_{0}+1\right)+A r \mathbf{B}\left(\alpha-q, \sigma_{1}+1\right)+B r \mathbf{B}\left(\alpha-q, \sigma_{2}+1\right)\right] \\
& \quad+M_{0}\left[C \int_{t_{1} / t_{2}}^{1}(1-w)^{\alpha-q-1} w^{\sigma_{0}} d w+A r \int_{t_{1} / t_{2}}^{1}(1-w)^{\alpha-q-1} w^{\sigma_{1}} d w\right. \\
& +
\end{aligned}
$$


Similarly, it can be shown that both

$$
\frac{\left|x_{1}-\int_{0}^{\infty} f\left(s, v(s), D_{0^{+}}^{p} v(s)\right) d s\right|}{\Gamma(\alpha-q)}
$$

and

$$
\left|x_{0}+b x_{1}-\int_{0}^{\infty} f\left(s, v(s), D_{0^{+}}^{p} v(s)\right) d s\right| \frac{\Gamma(\alpha-1)}{|\Gamma(\alpha-q-1)|}
$$

are uniformly bounded. Then

$$
\begin{aligned}
& \mid \frac{t_{1}^{2+q-\alpha}}{1+t_{1}^{\sigma+2}} \int_{0}^{t_{1}}\left(t_{1}-s\right)^{\alpha-q-1} f\left(s, v(s), D_{0^{+}}^{p} \nu(s)\right) d s \\
& \quad-\frac{t_{2}^{2+q-\alpha}}{1+t_{2}^{\sigma+2}} \int_{0}^{t_{2}}\left(t_{2}-s\right)^{\alpha-q-1} f\left(s, v(s), D_{0^{+}}^{p} \nu(s)\right) d s \mid \\
& \rightarrow 0 \quad \text { uniformly in } \Omega_{1} \text { as } t_{2} \rightarrow t_{1} .
\end{aligned}
$$

From (11) and (12), we infer that $F_{1}: \Omega_{1} \rightarrow Y$ is equicontinuous on a finite closed interval of $(0, \infty)$. Similarly, we can show that $F_{2}: \Omega_{2} \rightarrow X$ is equicontinuous on a finite closed interval on $(0, \infty)$.

Step 4. Now we prove that both $F_{1}: \Omega_{1} \rightarrow Y$ and $F_{2}: \Omega_{2} \rightarrow X$ are equiconvergent as $t \rightarrow 0$. By the assumption $(\mathrm{H})$, we have

$$
\begin{aligned}
& \left|\frac{t^{2-\alpha}}{1+t^{\sigma+2}}\left(F_{1} y\right)(t)-\frac{x_{0}+b x_{1}-\int_{0}^{\infty} f\left(s, y(s), D_{0^{+}}^{p} y(s)\right) d s}{a}\right| \\
& \leq \frac{1}{\Gamma(\alpha)} \frac{1}{1+t^{\sigma+2}}\left[C t^{2+\sigma_{0}} \int_{0}^{1}(1-w)^{\alpha-1} w^{\sigma_{0}} d w\right. \\
& \left.\quad+A t^{2+\sigma_{1}} \int_{0}^{1}(1-w)^{\alpha-1} w^{\sigma_{1}} d w+B t^{2+\sigma_{2}} \int_{0}^{1}(1-w)^{\alpha-1} w^{\sigma_{2}} d w\right] \\
& \quad+\frac{\left|x_{1}\right|}{\Gamma(\alpha)} \frac{t}{1+t^{\sigma+2}}+\left|x_{0}\right| \frac{t^{\sigma+2}}{1+t^{\sigma+2}} \\
& \rightarrow 0 \quad \text { uniformly in } \Omega_{1} \text { as } t \rightarrow 0 .
\end{aligned}
$$

Furthermore, for $\sigma_{i} \in(-1, \sigma)$, we have

$$
\begin{aligned}
& \left|\frac{t^{2+q-\alpha}}{1+t^{\sigma+2}} D_{0^{+}}^{q}\left(F_{1} v\right)(t)-\frac{x_{0}+b x_{1}-\int_{0}^{\infty} f\left(s, v(s), D_{0^{+}}^{p} v(s)\right) d s}{a} \frac{\Gamma(\alpha-1)}{\Gamma(\alpha-q-1)}\right| \\
& \leq \frac{1}{\Gamma(\alpha-q)}\left[C \frac{t^{2+\sigma_{0}}}{1+t^{\sigma+2}} \mathbf{B}\left(\alpha-q, \sigma_{0}+1\right)+A \frac{t^{2+\sigma_{1}}}{1+t^{\sigma+2}} \mathbf{B}\left(\alpha-q, \sigma_{1}+1\right)\right. \\
& \left.+B \frac{t^{2+\sigma_{2}}}{1+t^{\sigma+2}} \mathbf{B}\left(\alpha-q, \sigma_{2}+1\right)\right] \\
& +\frac{\left|x_{1}\right|+\left[C \Gamma\left(\sigma_{0}+1\right)+A \Gamma\left(\sigma_{1}+1\right)+B \Gamma\left(\sigma_{2}+1\right)\right]}{\Gamma(\alpha-q)} \frac{t}{1+t^{\sigma+2}} \\
& +\frac{\left|x_{0}+b x_{1}\right|+\left[C \Gamma\left(\sigma_{0}+1\right)+A \Gamma\left(\sigma_{1}+1\right)+B \Gamma\left(\sigma_{2}+1\right)\right]}{a} \frac{\Gamma(\alpha-1)}{\Gamma(\alpha-q-1)} \frac{t^{\sigma+2}}{1+t^{\sigma+2}} \\
& \rightarrow 0 \text { uniformly in } \Omega_{1} \text { as } t \rightarrow 0 \text {. }
\end{aligned}
$$


Hence $F_{1}: \Omega_{1} \rightarrow Y$ is equiconvergent as $t \rightarrow 0$. Similarly, we can prove that $F_{2}: \Omega_{2} \rightarrow Y$ is equiconvergent as $t \rightarrow 0$.

Step 5. Finally, we show that both $F_{1}: \Omega_{1} \rightarrow Y$ and $F_{2}: \Omega_{2} \rightarrow X$ are equiconvergent as $t \rightarrow \infty$. By the assumption $(\mathrm{H})$, we have

$$
\begin{aligned}
\left|\frac{t^{2-\alpha}}{1+t^{\sigma+2}}\left(F_{1} v\right)(t)\right| \leq & \frac{r}{\Gamma(\alpha)}\left[\frac{C t^{2+\sigma_{0}}}{1+t^{\sigma+2}} \int_{0}^{1}(1-w)^{\alpha-1} w^{\sigma_{0}} d w\right. \\
& \left.+\frac{A t^{2+\sigma_{1}}}{1+t^{\sigma+2}} \int_{0}^{1}(1-w)^{\alpha-1} w^{\sigma_{1}} d w+\frac{B t^{2+\sigma_{2}}}{1+t^{\sigma+2}} \int_{0}^{1}(1-w)^{\alpha-1} w^{\sigma_{2}} d w\right] \\
& +\frac{\left|x_{1}\right|}{\Gamma(\alpha)} \frac{t}{1+t^{\sigma+2}}+\frac{\left|x_{0}\right|}{1+t^{\sigma+2}} \\
\rightarrow & 0 \quad \text { uniformly in } \Omega_{1} \text { as } t \rightarrow \infty .
\end{aligned}
$$

Furthermore, for $\sigma_{i} \in(-1, \sigma)$, we have

$$
\begin{aligned}
\left|\frac{t^{2+q-\alpha}}{1+t^{\sigma+2}} D_{0^{+}}^{q}\left(F_{1} v\right)(t)\right| \\
\leq \frac{r}{\Gamma(\alpha-q)}\left[C \frac{t^{2+\sigma_{0}}}{1+t^{\sigma+2}} \mathbf{B}\left(\alpha-q, \sigma_{0}+1\right)+A \frac{t^{2+\sigma_{1}}}{1+t^{\sigma+2}} \mathbf{B}\left(\alpha-q, \sigma_{1}+1\right)\right. \\
\left.\quad+B \frac{t^{2+\sigma_{2}}}{1+t^{\sigma+2}} \mathbf{B}\left(\alpha-q, \sigma_{2}+1\right)\right] \\
\quad+\frac{\left|x_{1}\right|+r\left[C \Gamma\left(\sigma_{0}+1\right)+A \Gamma\left(\sigma_{1}+1\right)+B \Gamma\left(\sigma_{2}+1\right)\right]}{\Gamma(\alpha-q)} \frac{t}{1+t^{\sigma+2}} \\
\quad+\frac{\left|x_{0}+b x_{1}\right|+r\left[C \Gamma\left(\sigma_{0}+1\right)+A \Gamma\left(\sigma_{1}+1\right)+B \Gamma\left(\sigma_{2}+1\right)\right]}{a} \frac{\Gamma(\alpha-1)}{\Gamma(\alpha-q-1)} \frac{1}{1+t^{\sigma+2}} \\
\rightarrow 0 \quad \text { uniformly in } \Omega_{1} \text { as } t \rightarrow \infty .
\end{aligned}
$$

Hence $F_{1}: \Omega_{1} \rightarrow Y$ is equiconvergent as $t \rightarrow \infty$. Similarly, we can prove that $F_{2}: \Omega_{2} \rightarrow Y$ is equiconvergent as $t \rightarrow \infty$.

Thus, $F_{1}$ and $F_{2}$ are completely continuous. Hence $F$ is completely continuous.

Similarly, we can show that the results hold if $(G)$ holds. These complete the proofs.

\section{Main results}

In this section, we present the main results of the paper. For the sake of convenience, let us set

$$
\begin{aligned}
M_{1}= & \max \left\{\frac{A}{\Gamma(\alpha)} \mathbf{B}\left(\alpha, \sigma_{1}+1\right)+\frac{B}{\Gamma(\alpha)} \mathbf{B}\left(\alpha, \sigma_{2}+1\right)\right. \\
& +\left(\frac{1}{\Gamma(\alpha)}+\frac{1}{a}\right)\left(\frac{A}{k_{1}^{\sigma_{1}+1}} \Gamma\left(\sigma_{1}+1\right)+\frac{B}{k_{2}^{\sigma_{2}+1}} \Gamma\left(\sigma_{2}+1\right)\right), \\
& \frac{A}{\Gamma(\alpha-q)} \mathbf{B}\left(\alpha-q, \sigma_{1}+1\right)+\frac{B}{\Gamma(\alpha-q)} \mathbf{B}\left(\alpha-q, \sigma_{2}+1\right) \\
& \left.+\left(\frac{1}{\Gamma(\alpha-q)}+a \frac{\Gamma(\alpha-1)}{|\Gamma(\alpha-q-1)|}\right)\left(\frac{A}{k_{1}^{\sigma_{1}+1}} \Gamma\left(\sigma_{1}+1\right)+\frac{B}{k_{2}^{\sigma_{2}+1}} \Gamma\left(\sigma_{2}+1\right)\right)\right\},
\end{aligned}
$$




$$
\begin{aligned}
M_{2}= & \max \left\{\frac{A_{1}}{\Gamma(\beta)} \mathbf{B}\left(\beta, \mu_{1}+1\right)+\frac{B_{1}}{\Gamma(\beta)} \mathbf{B}\left(\beta, \mu_{2}+1\right)\right. \\
& +\left(\frac{1}{\Gamma(\beta)}+\frac{1}{c}\right)\left(\frac{A_{1}}{l_{1}^{\mu_{1}+1}} \Gamma\left(\mu_{1}+1\right)+\frac{B_{1}}{l_{2}^{\mu_{2}+1}} \Gamma\left(\mu_{2}+1\right)\right), \\
& \frac{A_{1}}{\Gamma(\beta-p)} \mathbf{B}\left(\beta-p, \mu_{1}+1\right)+\frac{B_{1}}{\Gamma(\beta-p)} \mathbf{B}\left(\beta-p, \mu_{2}+1\right) \\
& \left.+\left(\frac{1}{\Gamma(\beta-p)}+c \frac{\Gamma(\beta-1)}{|\Gamma(\beta-p-1)|}\right)\left(\frac{A_{1}}{l_{1}^{\mu_{1}+1}} \Gamma\left(\mu_{1}+1\right)+\frac{B_{1}}{l_{2}^{\mu_{2}+1}} \Gamma\left(\mu_{2}+1\right)\right)\right\} .
\end{aligned}
$$

Theorem 3.1 Suppose that $(\mathrm{H})$ holds. Then (5) has at least one solution $(x, y) \in X$ if

$$
\max \left\{M_{1}, M_{2}\right\}<1
$$

Proof Let $X \times Y$ be the Banach space equipped with the norm $\|\cdot\|$ (defined in Section 2). We seek the solutions of (5) by obtaining the fixed point of $F$ in $X \times Y$. Note that $F$ is well defined and completely continuous by Lemma 2.3 .

Let

$$
\begin{aligned}
\Psi(t)= & \frac{C}{\Gamma(\alpha)} \int_{0}^{t}(t-s)^{\alpha-1} s^{\sigma_{0}} e^{-k_{0} s} d s+\frac{x_{1}-C \int_{0}^{\infty} s^{\sigma_{0}} e^{-k_{0} s} d s}{\Gamma(\alpha)} t^{\alpha-1} \\
& +\frac{x_{0}+b x_{1}-C \int_{0}^{\infty} s^{\sigma_{0}} e^{-k_{0} s} d s}{a} t^{\alpha-2}, \\
\Phi(t)= & \frac{C_{1}}{\Gamma(\beta)} \int_{0}^{t}(t-s)^{\alpha-1} s^{\mu_{0}} e^{-l_{0} s} d s+\frac{y_{1}-C_{1} \int_{0}^{\infty} s^{\mu_{0}} e^{-l_{0} s} d s}{\Gamma(\alpha)} t^{\beta-1} \\
& +\frac{y_{0}+d y_{1}-C_{1} \int_{0}^{\infty} s^{\mu_{0}} e^{-l_{0} s} d s}{c} t^{\beta-2} .
\end{aligned}
$$

It is easy to show that $(\Psi, \Phi) \in X \times Y$. For $r>0$, we define

$$
M_{r}=\{(x, y) \in X \times Y:\|(x, y)-(\Psi, \Phi)\| \leq r\}
$$

For $(x, y) \in M_{r}$, we have $\|(x, y)-(\Psi, \Phi)\| \leq r$. Then

$$
\begin{aligned}
\|(x, y)\| & \leq\|(x, y)-(\Psi, \Phi)\|+\|(\Psi, \Phi)\| \leq r+\|(\Psi, \Phi)\|, \\
\|(x, y)\| & =\max \left\{\|x\|_{X},\|y\|_{Y}\right\} \leq r+\|(\Psi, \Phi)\| .
\end{aligned}
$$

Using the condition $(\mathrm{H})$ together with the method employed in Step 1 of the proof of Lemma 2.3, we find that

$$
\begin{aligned}
& \left|f\left(t, v_{n}(t), D_{0^{+}}^{p} v_{n}(t)\right)-C s^{\sigma_{0}} e^{-k_{0} s}\right| \\
& \quad \leq\left[A t^{\sigma_{1}} e^{-k_{1} t}+B t^{\sigma_{2}} e^{-k_{2} t}\right]\|(x, y)\|
\end{aligned}
$$

and

$$
\left|g\left(t, u_{n}(t), D_{0^{+}}^{q} u_{n}(t)\right)-C_{1} t^{\mu_{0}} e^{-l_{0} t}\right| \leq\left[A_{1} t^{\mu_{1}} e^{-l_{1} t}+B_{1} t^{\mu_{2}} e^{-l_{2} t}\right]\|(x, y)\| .
$$


Then

$$
\begin{aligned}
& \frac{t^{2-\alpha}}{1+t^{\sigma+2}}\left|\left(F_{1} y\right)(t)-\Psi(t)\right| \\
& \leq[r+\|(\Psi, \Phi)\|]\left[\frac{A}{\Gamma(\alpha)} \mathbf{B}\left(\alpha, \sigma_{1}+1\right)+\frac{B}{\Gamma(\alpha)} \mathbf{B}\left(\alpha, \sigma_{2}+1\right)\right. \\
& \left.\quad+\left(\frac{1}{\Gamma(\alpha)}+\frac{1}{a}\right)\left(\frac{A}{k_{1}^{\sigma_{1}+1}} \Gamma\left(\sigma_{1}+1\right)+\frac{B}{k_{2}^{\sigma_{2}+1}} \Gamma\left(\sigma_{2}+1\right)\right)\right] .
\end{aligned}
$$

Furthermore, we have

$$
\begin{aligned}
& \frac{t^{2+q-\alpha}}{1+t^{\sigma+2}}\left|D_{0^{+}}^{q}\left(F_{1} y\right)(t)-D_{0^{+}}^{q} \Psi(t)\right| \\
& \leq[r+\|(\Psi, \Phi)\|]\left[\frac{A}{\Gamma(\alpha-q)} \mathbf{B}\left(\alpha-q, \sigma_{1}+1\right)+\frac{B}{\Gamma(\alpha-q)} \mathbf{B}\left(\alpha-q, \sigma_{2}+1\right)\right. \\
& \left.\quad+\left(\frac{1}{\Gamma(\alpha-q)}+a \frac{\Gamma(\alpha-1)}{|\Gamma(\alpha-q-1)|}\right)\left(\frac{A}{k_{1}^{\sigma_{1}+1}} \Gamma\left(\sigma_{1}+1\right)+\frac{B}{k_{2}^{\sigma_{2}+1}} \Gamma\left(\sigma_{2}+1\right)\right)\right]
\end{aligned}
$$

Thus, it follows that

$$
\left\|F_{1} y-\Psi\right\|_{X} \leq[r+\|(\Psi, \Phi)\|] M_{1} .
$$

Similarly, one can obtain

$$
\left\|F_{2} x-\Phi\right\|_{Y} \leq[r+\|(\Psi, \Phi)\|] M_{2} .
$$

Hence

$$
\|F(x, y)-(\Psi, \Phi)\| \leq[r+\|(\Psi, \Phi)\|] \max \left\{M_{1}, M_{2}\right\}
$$

We choose

$$
r \geq \frac{\|(\Psi, \Phi)\| \max \left\{M_{1}, M_{2}\right\}}{1-\max \left\{M_{1}, M_{2}\right\}} .
$$

Then, for $(x, y) \in M_{r}$, we have

$$
\|F(x, y)-(\Psi, \Phi)\| \leq r
$$

Then the Schauder fixed point theorem implies that $F$ has a fixed point $(x, y) \in M_{r}$, which is a bounded solution of (5). The proof is complete.

Theorem 3.2 Suppose that (G) holds. Then (5) has at least one solution $(x, y) \in X \times Y$ if

$$
\frac{\|(\Psi, \Phi)\|^{1-\delta}(\delta-1)^{\delta-1}}{\delta^{\delta}} \geq \max \left\{M_{1}, M_{2}\right\}
$$


Proof With $\Psi$ and $\Phi$ defined in the proof of Theorem 3.1, it is easy to show that $(\Psi, \Phi) \in$ $X \times Y$. For $(x, y) \in M_{r}$ (defined in the proof of Theorem 3.1), using (G) and the method of the proof for Theorem 3.1, we find that

$$
\|F(x, y)-(\Psi, \Phi)\| \leq[r+\|(\Psi, \Phi)\|]^{\delta} \max \left\{M_{1}, M_{2}\right\}
$$

Let $r=r_{0}=\frac{\|(\Psi, \Phi)\|}{\delta-1}, \delta>1$. Then

$$
\frac{r_{0}}{\left(r_{0}+\|(\Psi, \Phi)\|\right)^{\delta}}=\frac{\|(\Psi, \Phi)\|^{1-\delta}(\delta-1)^{\delta-1}}{\delta^{\delta}} \geq \max \left\{M_{1}, M_{2}\right\} .
$$

Thus, for $(x, y) \in M_{r_{0}}$, we have

$$
\|F(x, y)-(\Psi, \Phi)\| \leq r_{0}
$$

Hence, we obtain a bounded subset $M_{r_{0}} \subseteq X \times Y$ such that $T\left(M_{r_{0}}\right) \subseteq M_{r_{0}}$. In consequence, by the Schauder fixed point theorem, $F$ has a fixed point $(x, y) \in M_{r_{0}}$. Hence, $(x, y)$ is a bounded solution of (5). This completes the proof.

\section{An example}

Consider the fractional boundary value problem given by

$$
\left\{\begin{array}{l}
D_{0^{+}}^{\frac{3}{2}} x(t)=C t^{-\frac{3}{4}} e^{-t}+A \frac{t^{-\frac{1}{2}} e^{-2 t}}{1+t^{\frac{3}{2}}} y(t)+B \frac{\frac{9}{10} e^{-3 t}}{1+t^{\frac{3}{2}}} D_{0^{+}}^{\frac{6}{5}} y(t), \quad t \in(0, \infty), \\
D_{0^{+}}^{\frac{7}{4}} y(t)=C t^{-\frac{3}{4}} e^{-t}+A \frac{t^{-\frac{1}{4}} e^{-2 t}}{1+t^{\frac{3}{2}}} x(t)+B \frac{\frac{7}{10} e^{-3 t}}{1+t^{\frac{3}{2}}} D_{0^{+}}^{\frac{3}{4}} x(t), \quad t \in(0, \infty) \\
\lim _{t \rightarrow 0} t^{2-\alpha} x(t)-\lim _{t \rightarrow 0} D_{0^{+}}^{\alpha-1} x(t)=x_{0} \\
\lim _{t \rightarrow 0} t^{2-\beta} y(t)-\lim _{t \rightarrow 0} D_{0^{+}}^{\beta-1} x(t)=y_{0} \\
\lim _{t \rightarrow \infty} D_{0^{+}}^{\alpha-1} x(t)=x_{1} \\
\lim _{t \rightarrow \infty} D_{0^{+}}^{\beta-1} x(t)=y_{1}
\end{array}\right.
$$

where $A, B, C>0$ and $x_{0}, x_{1}, y_{0}, y_{1} \in R$ are constants, $\alpha=\frac{3}{2}, \beta=\frac{7}{4}, p=\frac{6}{5}, q=\frac{3}{4}, a=b=c=$ $d=1$ and

$$
\begin{aligned}
& f(t, x, y)=C t^{-\frac{3}{4}} e^{-t}+A \frac{t^{-\frac{1}{2}} e^{-2 t}}{1+t^{\frac{3}{2}}} x+B \frac{t^{\frac{9}{10}} e^{-3 t}}{1+t^{\frac{3}{2}}} y, \\
& g(t, x, y)=C t^{-\frac{3}{4}} e^{-t}+A \frac{t^{-\frac{1}{4}} e^{-2 t}}{1+t^{\frac{3}{2}}} x+B \frac{t^{\frac{7}{10}} e^{-3 t}}{1+t^{\frac{3}{2}}} y .
\end{aligned}
$$

Note that $p \in(\beta-1, \beta)$ and $q \in(\alpha-1, \alpha)$.

Choose $\sigma=-\frac{1}{2}>\max \{q-\alpha, p-\beta\}, \sigma_{0}=\mu_{0}=-\frac{3}{4}, \sigma_{1}=\mu_{1}=-\frac{3}{4}, \sigma_{2}=\mu_{2}=-\frac{11}{20}, k_{0}=l_{0}=1$, $k_{1}=l_{1}=2, k_{2}=l_{2}=3$. One sees that $\sigma_{i}, \mu_{i} \in(-1, \sigma), k_{i}>0, l_{i}>0(i=0,1,2)$.

Thus,

$$
\begin{aligned}
& f\left(t, \frac{1+t^{\frac{3}{2}}}{t^{\frac{1}{4}}} x, \frac{1+t^{\frac{3}{2}}}{t^{\frac{29}{20}}} y\right)=C t^{-\frac{3}{4}} e^{-t}+A t^{-\frac{3}{4}} e^{-2 t} x+B t^{-\frac{11}{20}} e^{-3 t} y, \\
& g\left(t, \frac{1+t^{\frac{3}{2}}}{t^{\frac{1}{2}}} x, \frac{1+t^{\frac{3}{2}}}{t^{\frac{5}{4}}} y\right)=C t^{-\frac{3}{4}} e^{-t}+A t^{-\frac{3}{4}} e^{-2 t} x+B t^{-\frac{11}{20}} e^{-3 t} y .
\end{aligned}
$$


It is easy to show that $(\mathrm{H})$ holds. By direct computation, we get

$$
\begin{aligned}
M_{1}= & \max \left\{\frac{\mathbf{B}(3 / 2,1 / 4)}{\Gamma(3 / 2)} A+\frac{\mathbf{B}(3 / 2,9 / 20)}{\Gamma(3 / 2)} B+\frac{1+\Gamma(3 / 2)}{\Gamma(3 / 2)}\left(\frac{\Gamma(1 / 4)}{2^{1 / 4}} A+\frac{\Gamma(9 / 20)}{3^{9 / 20}} B\right),\right. \\
& \frac{\mathbf{B}(3 / 4,1 / 4)}{\Gamma(3 / 4)} A+\frac{\mathbf{B}(3 / 4,9 / 20)}{\Gamma(3 / 4)} B \\
& \left.+\left(\frac{1}{\Gamma(3 / 4)}+\frac{\Gamma(1 / 2)}{|\Gamma(-1 / 4)|}\right)\left(\frac{\Gamma(1 / 4)}{2^{1 / 4}} A+\frac{\Gamma(9 / 20)}{3^{9 / 20}} B\right)\right\}, \\
M_{2}= & \max \left\{\frac{\mathbf{B}(7 / 4,1 / 4)}{\Gamma(7 / 4)} A+\frac{\mathbf{B}(7 / 4,9 / 20)}{\Gamma(7 / 4)} B+\frac{1+\Gamma(7 / 4)}{\Gamma(7 / 4)}\left(\frac{\Gamma(1 / 4)}{2^{1 / 4}} A+\frac{\Gamma(9 / 20)}{3^{9 / 20}} B\right),\right. \\
& \frac{\mathbf{B}(11 / 20,1 / 4)}{\Gamma(11 / 20)} A+\frac{\mathbf{B}(11 / 20,9 / 20)}{\Gamma(11 / 20)} B \\
& \left.+\left(\frac{1}{\Gamma(11 / 20)}+\frac{\Gamma(3 / 4)}{|\Gamma(-9 / 20)|}\right)\left(\frac{\Gamma(1 / 4)}{2^{1 / 4}} A+\frac{\Gamma(9 / 20)}{3^{9 / 20}} B\right)\right\} .
\end{aligned}
$$

Thus, Theorem 3.1 applies and BVP (16) has at least one solution $(x, y) \in X \times Y$ if $\max \left\{M_{1}, M_{2}\right\}<1$. This solution satisfies that

$$
\frac{t^{\frac{1}{2}}}{1+t^{\frac{3}{2}}}|x(t)|, \quad \frac{t^{\frac{1}{4}}}{1+t^{\frac{3}{2}}}|y(t)|, \quad \frac{t^{\frac{5}{4}}}{1+t^{\frac{3}{2}}}\left|D_{0^{+}}^{\frac{3}{4}} x(t)\right|, \quad \frac{t^{\frac{29}{20}}}{1+t^{\frac{3}{2}}}\left|D_{0^{+}}^{\frac{6}{5}} y(t)\right|
$$

are bounded on $(0, \infty)$.

Remark 4.1 It is easy to see that $\max \left\{M_{1}, M_{2}\right\}<1$ holds for sufficiently small $A>0$ and $B>0$. One notes that $\alpha=\frac{3}{2}, \beta=\frac{7}{4}, p=\frac{6}{5}, q=\frac{3}{4}$ in the mentioned example. It is easy to see that $\alpha-q \geq 1$ and $\beta-p \geq 1$ do not hold. Hence theorems in [34,35] cannot be applied to solve this example.

\section{Competing interests}

The authors declare that they have no competing interests.

\section{Authors' contributions}

Each of the authors, YL, BA and RPA, contributed to each part of this study equally and read and approved the final version of the manuscript.

\section{Author details}

${ }^{1}$ Department of Mathematics, Guangdong University of Business Studies, Guangzhou, 510000, P.R. China. ${ }^{2}$ Department of Mathematics, Faculty of Science, King Abdulaziz University, P.O. Box 80203, Jeddah, 21589, Saudi Arabia. ${ }^{3}$ Department of Mathematics, Texas A\&M University-Kingsville, University Blvd., Kingsville, TX 78363-8202, USA.

\section{Acknowledgements}

The first author was supported by the Natural Science Foundation of Guangdong province (No: S2011010001900) and the Guangdong Higher Education Foundation for High-level talents. This paper was funded by King Abdulaziz University, under grant No. (130-1-1433/HiCi). The authors, therefore, acknowledge technical and financial support of KAU.

Received: 7 July 2012 Accepted: 5 February 2013 Published: 1 March 2013

\section{References}

1. Miller, KS, Ross, B: An Introduction to the Fractional Calculus and Fractional Differential Equation. Wiley, New York (1993)

2. Samko, SG, Kilbas, AA, Marichev, Ol: Fractional Integral and Derivative. Theory and Applications. Gordon \& Breach, New York (1993)

3. Kilbas, AA, Srivastava, HM, Trujillo, JJ: Theory and Applications of Fractional Differential Equations. North-Holland Mathematics Studies, vol. 204. Elsevier, Amsterdam (2006) 
4. Sabatier, J, Agrawal, OP, Machado, JT (eds.): Advances in Fractional Calculus: Theoretical Developments and Applications in Physics and Engineering. Springer, Dordrecht (2007)

5. Lakshmikantham, V, Leela, S, Devi, JV: Theory of Fractional Dynamic Systems. Cambridge Academic Publishers, Cambridge (2009)

6. Baleanu, D, Diethelm, K, Scalas, E, Trujillo, JJ: Fractional Calculus Models and Numerical Methods. Series on Complexity, Nonlinearity and Chaos. World Scientific, Boston (2012)

7. Podlubny, I: Geometric and physical interpretation of fractional integral and fractional differentiation. Fract. Calc. Appl. Anal. 5, 367-386 (2002)

8. Heymans, N, Podlubny, l: Physical interpretation of initial conditions for fractional differential equations involving Riemann-Liouville fractional derivatives on the half line. Rheol. Acta 45, 765-771 (2006)

9. West, BJ, Bologna, M, Grigolini, P: Physics of Fractal Operators. Springer, New York (2003)

10. Zhang, S: Monotone iterative method for initial value problem involving Riemann-Liouville fractional derivatives. Nonlinear Anal. 71, 2087-2093 (2009)

11. Agarwal, RP, Benchohra, M, Hamani, S: A survey on existence result for boundary value problems of nonlinear fractional differential equations and inclusions. Acta Appl. Math. 109, 973-1033 (2010)

12. Wei, Z, Li, Q, Che, J: Initial value problems for fractional differential equations involving Riemann-Liouville sequential fractional derivative. J. Math. Anal. Appl. 367, 260-272 (2010)

13. Agarwal, RP, Zhou, Y, He, Y: Existence of fractional neutral functional differential equations. Comput. Math. Appl. 59, 1095-1100 (2010)

14. Bai, Z: On positive solutions of a nonlocal fractional boundary value problem. Nonlinear Anal. 72, 916-924 (2010)

15. Hernández, E, O'Regan, D, Balachandran, K: On recent developments in the theory of abstract differential equations with fractional derivatives. Nonlinear Anal. 73, 3462-3471 (2010)

16. Baleanu, D, Mustafa, OG, Agarwal, RP: An existence result for a superlinear fractional differential equation. Appl. Math. Lett. 23, 1129-1132 (2010)

17. Ahmad, B, Nieto, JJ: Anti-periodic fractional boundary value problems. Comput. Math. Appl. 62, 1150-1156 (2011)

18. Ahmad, B, Nieto, J: Riemann-Liouville fractional integro-differential equations with fractional nonlocal integral boundary conditions. Bound. Value Probl. 2011, 36 (2011)

19. Ahmad, B, Ntouyas, SK: A four-point nonlocal integral boundary value problem for fractional differential equations of arbitrary order. Electron. J. Qual. Theory Differ. Equ. 2011, 22 (2011)

20. Ahmad, B, Nieto, JJ, Alsaedi, A, El-Shahed, M: A study of nonlinear Langevin equation involving two fractional orders in different intervals. Nonlinear Anal., Real World Appl. 13, 599-606 (2012)

21. Ahmad, B, Nieto, JJ: Riemann-Liouville fractional differential equations with fractional boundary conditions. Fixed Point Theory 13, 329-336 (2012)

22. Arara, A, Benchohra, M, Hamidi, N, Nieto, JJ: Fractional order differential equations on an unbounded domain. Nonlinear Anal. 72, 580-586 (2010)

23. Zhao, X, Ge, W: Unbounded positive solutions for a fractional boundary value problem on the half-lines. Acta Appl. Math. 109, 495-505 (2010)

24. Li, X, Jia, M: Multiple solutions of nonlocal boundary value problems for fractional differential equations on the half line. Electron. J. Qual. Theory Differ. Equ. 2011, 56 (2011)

25. Su, X, Zhang, S: Unbounded solutions to a boundary value problem of fractional order on the half line. Comput. Math. Appl. 61, 1079-1087 (2011)

26. Su, X: Solutions to boundary value problem of fractional order on unbounded domains in a Banach space. Nonlinear Anal. 74, 2844-2852 (2011)

27. Liang, S, Zhang, J: Existence of multiple positive solutions for $m$-point fractional boundary value problems on an infinite interval. Math. Comput. Model. 54, 1334-1346 (2011)

28. Agarwal, RP, Benchohra, M, Hamidi, N, Pinelas, S: Boundary value problems for differential equations involving Riemann-Liouville fractional derivative on the half line. Dyn. Contin. Discrete Impuls. Syst., Ser. A Math. Anal. 18, 235-244 (2011)

29. Wang, G, Ahmad, B, Zhang, L: A coupled system of nonlinear fractional differential equations with multi-point fractional boundary conditions on an unbounded domain. Abstr. Appl. Anal. 2012, Article ID 248709 (2012)

30. Chen, $Y, A n, H$ : Numerical solutions of coupled Burgers equations with time and space fractional derivatives. Appl. Math. Comput. 200, 87-95 (2008)

31. Gafiychuk, V, Datsko, B, Meleshko, V: Mathematical modeling of time fractional reaction-diffusion systems. J. Comput. Appl. Math. 220, 215-225 (2008)

32. Lazarevic, MP: Finite time stability analysis of $P D^{\alpha}$ fractional control of robotic time-delay systems. Mech. Res. Commun. 33, 269-279 (2006)

33. Gafiychuk, V, Datsko, B, Meleshko, V, Blackmore, D: Analysis of the solutions of coupled nonlinear fractional reaction-diffusion equations. Chaos Solitons Fractals 41, 1095-1104 (2009)

34. Su, X: Boundary value problem for a coupled system of nonlinear fractional differential equations. Appl. Math. Lett. 22, 64-69 (2009)

35. Ahmad, B, Nieto, J: Existence results for a coupled system of nonlinear fractional differential equations with three-point boundary conditions. Comput. Math. Appl. 58, 1838-1843 (2009)

36. Goodrich, CS: Existence of a positive solution to systems of differential equations of fractional order. Comput. Math. Appl. 62, 1251-1268 (2011)

37. Ntouyas, SK, Obaid, M: A coupled system of fractional differential equations with nonlocal integral boundary conditions. Adv. Differ. Equ. 2012, 130 (2012)

doi:10.1186/1687-1847-2013-46

Cite this article as: Liu et al.: Existence of solutions for a coupled system of nonlinear fractional differential equations with fractional boundary conditions on the half-line. Advances in Difference Equations 2013 2013:46. 